\title{
Postoperative solitary liver metastasis from esophageal squamous cell carcinoma achieving a clinical complete response to chemotherapy with cisplatin and 5-fluorouracil followed by stereotactic body radiotherapy: A case report
}

\author{
SHUICHI FUKUDA ${ }^{1}$, KAORU OKAJIMA ${ }^{2}$, KAORU OKADA ${ }^{1}$, KOHEI FUKUDA ${ }^{2}$, \\ TOMOKO WAKASA ${ }^{3}$, TOMOYUKI TSUJIMOTO ${ }^{1}$, ATSUSHI GAKUHARA ${ }^{1}$, HIDEO TOMIHARA ${ }^{1}$, \\ KATSUYA OHTA $^{1}$, KOTARO KITANI ${ }^{1}$, KAZUHIKO HASHIMOTO ${ }^{1}$, HAJIME ISHIKAWA ${ }^{1}$, \\ JIN-ICHI HIDA ${ }^{1}$, MASAO YUKAWA ${ }^{1}$, YOSHIO OHTA ${ }^{3}$ and MASATOSHI INOUE ${ }^{1}$ \\ Departments of ${ }^{1}$ Gastroenterological Surgery, ${ }^{2}$ Radiology and ${ }^{3}$ Pathology, \\ Kindai University Nara Hospital, Ikoma, Nara 630-0293, Japan
}

Received January 12, 2021; Accepted April 16, 2021

DOI: $10.3892 / \mathrm{mco} .2021 .2292$

\begin{abstract}
Treatment strategies for distant organ metastasis have changed markedly since the concept of oligometastasis was introduced. The perception that distant organ metastasis is a systemic disease and not eligible for local therapy is now a thing of the past. Therefore, the present report details a case of postoperative solitary liver metastasis from esophageal squamous cell carcinoma (ESCC), which achieved a clinical complete response to chemotherapy with cisplatin and 5-fluorouracil (5-FU) followed by stereotactic body radiotherapy (SBRT). A 76-year-old male patient underwent esophagectomy for lower thoracic ESCC. At 7 months after surgery, abdominal CT revealed a solitary hypovascular mass, $28 \mathrm{~mm}$ in size, in segment 7 of the liver. After three courses of chemotherapy with cisplatin and 5-FU, abdominal CT revealed that the liver mass had shrunk to $7 \mathrm{~mm}$ in size. SBRT was then administered with a $6 \mathrm{MV}$ X-ray beam generated by a linear accelerator. A total dose of $50 \mathrm{~Gy}$ was given in 5 fractions of $10 \mathrm{~Gy}$ to the liver mass. At 1 month after SBRT, abdominal CT revealed that the liver mass had disappeared. The patient received no further adjuvant chemotherapy and had no recurrence at 18 months after diagnosis of liver metastasis and 13 months after SBRT.
\end{abstract}

Correspondence to: Dr Shuichi Fukuda, Department of Gastroenterological Surgery, Kindai University Nara Hospital, 1248-1 Otoda-cho, Ikoma, Nara 630-0293, Japan

E-mail: s.f4911@nifty.com

Key words: esophageal cancer, liver metastases, oligometastasis, oligorecurrence, stereotactic ablative radiotherapy

\section{Introduction}

Due to the progression of neoadjuvant treatment strategies and surgical procedures, 3- and 5-year survival rates of patients who underwent esophagectomy with no residual tumors improved to 71.4 and $62.8 \%$, respectively (1). However, recurrence still occurs in $28-56 \%$ of patients who underwent radical resection of esophageal cancer, with a median time to recurrence of 10-12 months $(2,3)$. The median survival time after recurrence remains poor at 8.2 months (4). Recurrent esophageal cancer is considered a systemic disease, for which chemotherapy with cisplatin and 5-fluorouracil (5-FU) is used as the standard therapy (2). In a phase II study reported by Bleiberg et al (5), chemotherapy with cisplatin and 5-FU yielded a response rate of $35 \%$ and a median survival time of 7.6 months. In another phase II study reported by Iizuka et al (6), chemotherapy with cisplatin and 5-FU yielded a response rate of $35.9 \%$ and a median survival time of 9.5 months for patients who responded to the treatment.

Oligometastasis is a disease concept defined as a limited number of systemic metastatic tumors, usually characterized by fewer than five metastases, where curative strategies may be effective $(7,8)$. Recent reports have demonstrated that local therapy, such as resection, radiofrequency ablation (RFA), and radiotherapy, for oligometastasis from esophageal cancer can have a promising long-term prognosis (8-11). Regarding RFA, Baba et al (11) reported that the percutaneous computed tomography (CT)-guided RFA for pulmonary metastases from esophageal squamous cell carcinoma (ESCC) yielded $83 \%$ of local control of ablated tumor lasting for at least 1 year. The predicted 1- and 2-year overall survival rates after lung RFA were 77.8 and $62.2 \%$, respectively. The treatment strategy for recurrent esophageal cancer needs to be considered individually according to the extent of recurrence, recurrent site, and recurrent pattern (2). 

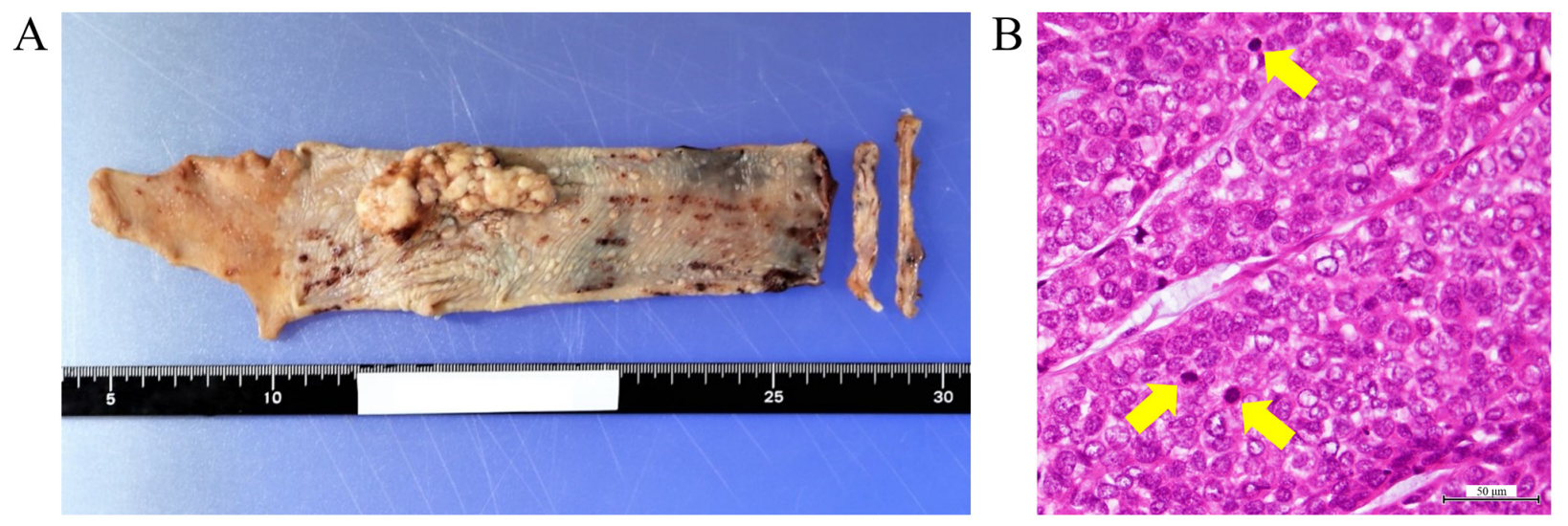

Figure 1. Resected specimen of esophageal cancer. (A) Gross appearance of the surgically resected specimen. (B) Histopathological findings of the resected specimen revealed a poorly differentiated squamous cell carcinoma. Keratinization was poor, and the mitotic count was $>10$ per high-power field. The arrows indicate mitoses. Scale bar, $50 \mu \mathrm{m}$.

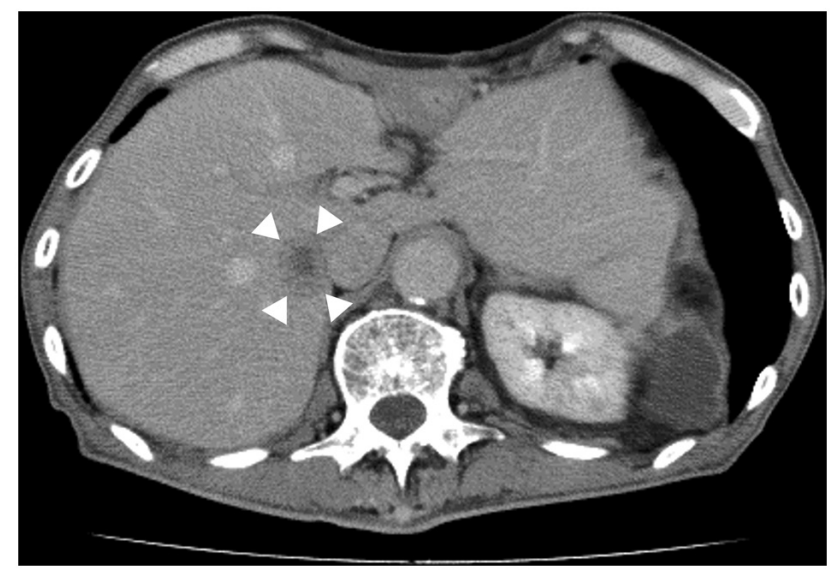

Figure 2. At 7 months after surgery, abdominal contrast-enhanced CT revealed a solitary hypovascular mass in segment 7 of the liver. The mass was $28 \mathrm{~mm}$ in size and was located adjacent to the inferior vena cava (arrowheads).

Stereotactic body radiotherapy (SBRT) has been identified as a modern radiation technique that delivers high doses of radiation to the target, while limiting the doses to the surrounding healthy tissue (12). SBRT for oligometastatic cancer has been reported to demonstrate good disease control, with acceptable rates of acute and late grade 3 or higher toxicities $(13,14)$. However, even among those cases, a clinical complete response of liver oligometastasis from ESCC is extremely rare. Here we present a case of a patient with postoperative solitary liver metastasis from ESCC who achieved a clinical complete response to chemotherapy with cisplatin and 5-fluorouracil followed by SBRT.

\section{Case report}

A 76-year-old man underwent esophagectomy with gastric tube reconstruction through the retrosternal route with cervical anastomosis for lower thoracic ESCC. The patient was diagnosed with T1bN0M0, stage IB according to the Union for International Cancer Control TNM classification of malignant tumors, 8th edition. The histopathological findings confirmed a poorly differentiated squamous cell carcinoma (Fig. 1). The

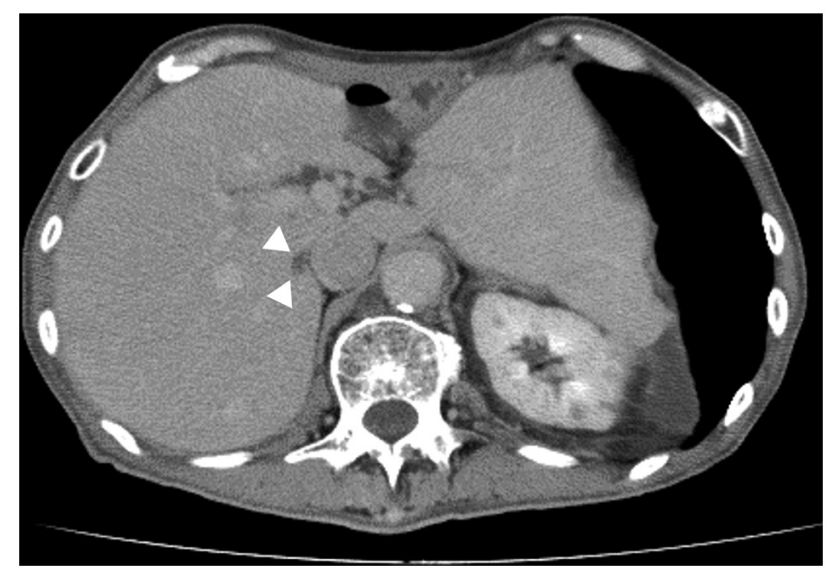

Figure 3. After three courses of chemotherapy, abdominal contrast-enhanced $\mathrm{CT}$ revealed that the liver mass had shrunk to $7 \mathrm{~mm}$ in size (arrowheads), with no evidence of further metastatic lesions. resected margins were determined to be free of tumor cells. No lymphatic invasion was observed; however, slight venous invasion was detected.

Four months after surgery, abdominal contrast-enhanced CT revealed no recurrence. Tumor markers were within the normal ranges: Fragment of cytokeratin subunit 19 (CYFRA 21-1), $2.9 \mathrm{ng} / \mathrm{ml}$ (normal level, $<3.5 \mathrm{ng} / \mathrm{ml}$ ) and squamous cell carcinoma antigen (SCC-Ag), $1.4 \mathrm{ng} / \mathrm{ml}$ (normal level, $<1.5 \mathrm{ng} / \mathrm{ml}$ ). However, 7 months after surgery, abdominal contrast-enhanced CT revealed a solitary hypovascular mass in segment 7 of the liver (Fig. 2). The mass was $28 \mathrm{~mm}$ in size and was located adjacent to the inferior vena cava. No segmental enhancement of the liver parenchyma around the liver mass at the early phase was observed, which was a characteristic CT finding of liver abscess. Furthermore, no enhancement of the liver mass and washout at the early and late phases, respectively, were observed, which were characteristic CT findings of hepatocellular carcinoma. Laboratory results showed no inflammation. The patient was negative for both hepatitis B virus surface antigen and hepatitis $\mathrm{C}$ virus antibody. Therefore, the possibility that the liver mass was liver abscess or hepatocellular carcinoma was ruled out. The risk of esophageal cancer 


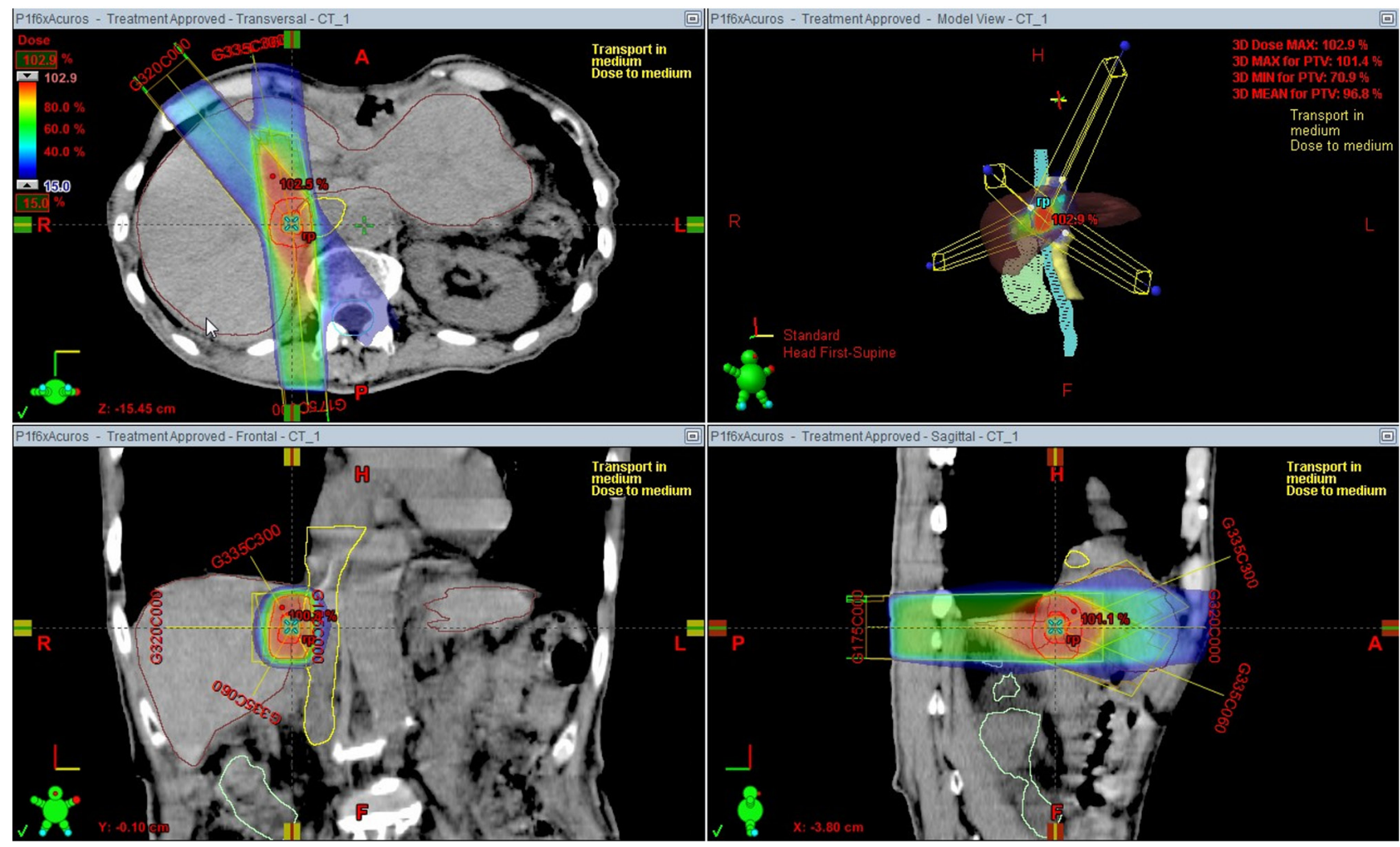

Figure 4. Simulation image of irradiation. A total dose of 50 Gy was given in 5 fractions of 10 Gy to the liver mass.

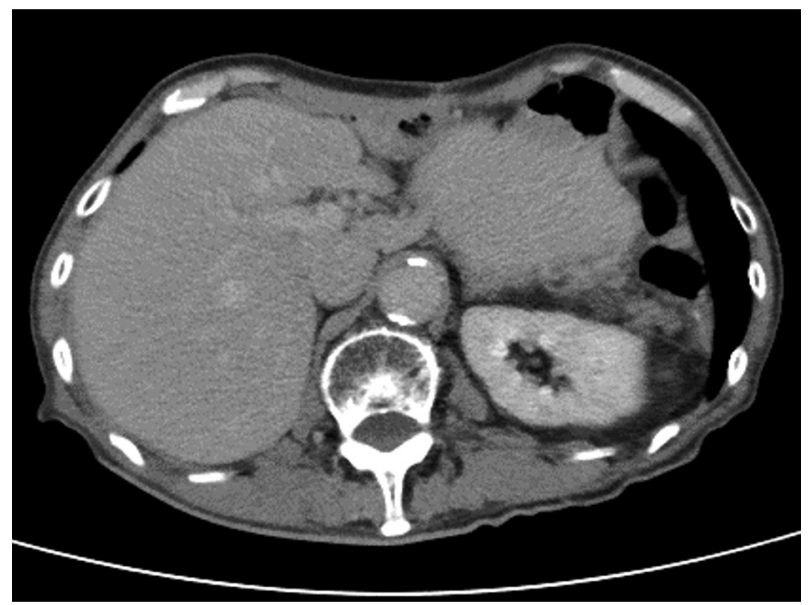

Figure 5. At 1 month after stereotactic body radiotherapy, abdominal contrast-enhanced CT revealed that the liver mass had disappeared.

recurrence was considered high due to the pathological findings of poorly differentiated squamous cell carcinoma and venous invasion. Regarding tumor markers, CYFRA 21-1 was within the normal range $(2.9 \mathrm{ng} / \mathrm{ml})$; however, SCC-Ag was slightly elevated $(2.1 \mathrm{ng} / \mathrm{ml})$. Given these results, the patient was diagnosed with postoperative solitary liver metastasis. Considering the early postoperative recurrence and rapid growth within 3 months, it was determined that systemic chemotherapy was necessary.

The patient then underwent chemotherapy consisting of cisplatin at $75 \mathrm{mg} / \mathrm{m}^{2}$ administered by rapid intravenous infusion on day 1 and $5-\mathrm{FU}$ at $1,000 \mathrm{mg} / \mathrm{m}^{2}$ administered by continuous intravenous infusion on days 1-5, separated by a 4 -week interval. The treatment was well tolerated, with no grade 3 or higher adverse events. After 3 courses of chemotherapy, abdominal contrast-enhanced CT revealed that the liver mass has shrunk to $7 \mathrm{~mm}$ in size, with no evidence of further metastatic lesions (Fig. 3). Gd-EOB-DTPA-enhanced magnetic resonance imaging has also confirmed the reduction in size and absence of any no other liver metastases. After discussion at a multidisciplinary cancer conference, SBRT at the liver mass was planned.

SBRT was administered with a 6 MV X-ray beam generated by a linear accelerator (Clinac iX; Varian Medical Systems). A total dose of 50 Gy was given in 5 fractions of 10 Gy to the liver mass (Fig. 4). The patient did not develop any acute or late toxicities. One month after SBRT, abdominal contrast-enhanced CT revealed that the liver mass has disappeared (Fig. 5). Furthermore, positron emission tomography revealed no significant accumulation of ${ }^{18} \mathrm{~F}$-fluorodeoxyglucose. Thus, a clinical complete response of the liver metastasis was achieved. The patient received no further adjuvant chemotherapy and had no recurrence at 18 months after diagnosis of liver metastasis and 13 months after SBRT.

\section{Discussion}

In this present case, a patient achieved a clinical complete response to postoperative solitary liver metastasis from ESCC with a combination of systemic therapy with chemotherapy and local therapy with SBRT. Esophageal cancer has been determined to be an aggressive and relatively common cancer worldwide; it is the sixth leading cause of cancer-related 
mortality and the eighth most common cancer worldwide (15). The prognosis is generally very poor despite recent advances in multidisciplinary treatment if distant organ metastasis occurs after curative treatment for esophageal cancer. Thus, there has been an urgent need to establish an effective treatment strategy for recurrent esophageal cancer. To the best of our knowledge, this present case is the first reported in the English literature of liver metastasis in an ESCC patient achieving a clinical complete response to chemotherapy followed by SBRT.

Treatment strategies for distant organ metastasis have changed dramatically since the introduction of the concept of oligometastasis. The perception that distant organ metastasis is a systemic disease and not eligible for local therapy is now a thing of the past. In general, chemotherapy works throughout the whole body, but it appears to be marginally effective only in high sensitivity cases (16). In advanced/recurrent ESCC patients, the response rate of cisplatin and 5-FU is $35-35.9 \%$, but the evidence for prolonged survival remains unclear, and it is usually administered with palliative intent $(2,5,6)$. On the other hand, local therapy works at the targeted site and can be curative, if treated radically. In this present case, the recurrence was solitary, but because of the early postoperative recurrence and rapid growth within 3 months, we were concerned about the aggressiveness of the tumor growth and considered the possibility of rapid multiple liver metastases and metastasis to other sites. Therefore, we chose systemic therapy with chemotherapy instead of local therapy. Fortunately, the tumor shrank and no additional metastases were observed. We then chose SBRT, which has a high local control effect, and it resulted in remission the liver metastasis. Our treatment strategy was consistent with Morinaga et al (17) who reported that chemotherapy with local therapy, such as resection, RFA, and radiotherapy, is a promising treatment modality for patients with oligometastatic recurrence after curative resection of ESCC.

Ohkura et al (10) reported that the overall survival rate was significantly better for patients who underwent resection of oligometastasis from esophageal cancer than for those who did not (3-year survival rates, 64.3 vs. 10\%, respectively; 5 -year survival rates, 55.6 vs. $10 \%$, respectively). Alternatively, SBRT is a radiation technique that enables irradiation of a small target in the body using a high dose. SBRT is useful in terms of quality of life because it is deemed noninvasive and has a short treatment time in addition to its curative potential. Rusthoven et al (18) reported excellent results of a multi-institutional phase I/II trial of SBRT for liver metastases. It reported at a median follow-up of 16 months actuarial in-field local control rates at 1 and 2 years of 95 and $92 \%$, respectively. Among lesions $<3 \mathrm{~cm}, 2$-year local control was $100 \%$. Furthermore, only $2 \%$ of patients experienced grade 3 or higher toxicities. Resection of metastasis is invasive, and there are many cases where surgery is not indicated due to lack of surgical tolerance or patient's will. Thus, SBRT can be a good treatment option, especially when resection of metastases is not applicable.

Recently, a consensus recommendation by the European Society for Radiotherapy and Oncology and European Organization for Research and Treatment of Cancer (ESTRO-EORTC) suggested dividing oligometastatic disease into synchronous (the interval time from initial diagnosis to metastasis within 6 months) and metachronous (the interval time from initial diagnosis to metastasis more than 6 months) $(9,19)$. Furthermore, metachronous oligometastatic disease in patients not under active systemic therapy is classified as metachronous oligorecurrence (19). Thus, this present case falls into this classification. Whether the treatment strategy for metachronous oligorecurrence is a local therapy alone, systemic therapy alone, or combination of local and systemic therapies has not yet been established. In this present case, liver oligometastasis from ESCC was successfully treated with systemic therapy using chemotherapy and subsequent local therapy with SBRT. We think it makes sense to proceed with systemic therapy before starting local therapy, considering the possibility of microscopic metastases to liver and other sites. In general, there is a very poor prognosis for esophageal cancer patients with recurrence within 12 months after radical esophagectomy $(4,20)$. Furthermore, liver recurrence is often associated with shortened survival period (4). Nevertheless, successful treatment was achieved in our case of an early postoperative liver metastasis from ESCC.

In conclusion, this present report detailed the case of a patient achieving a clinical complete response to postoperative solitary liver metastasis from ESCC by chemotherapy with cisplatin and 5-FU followed by SBRT. As this was a single-patient case report, our findings need to be confirmed by accumulating prospective evidence from more patients. However, these current findings provide important information that can contribute to the development of a treatment strategy for liver oligometastasis from ESCC.

\section{Acknowledgements}

Not applicable.

\section{Funding}

No funding was received.

\section{Availability of data and materials}

The datasets used and/or analyzed during the current study are available from the corresponding author on reasonable request.

\section{Authors' contributions}

SF conceived and designed the study. SF, KOkaj, KF, TW and YO acquired the data. SF, KOkad, TT, AG, HT, KOh, $\mathrm{KK}, \mathrm{KH}, \mathrm{HI}, \mathrm{JIH}, \mathrm{MY}$ and MI analyzed and interpreted the data. SF drafted the manuscript. SF, KOkaj and TW revised the manuscript critically for important intellectual content. SF, KOkaj and TW are responsible for confirming the authenticity of all the raw data. All authors read and approved the final manuscript.

\section{Ethics approval and consent to participate}

The present study was approved by the Ethics Committee of the Kindai University Nara Hospital (approval no. 20-25; Nara, Japan). 


\section{Patient consent for publication}

Written informed consent for the publication of data and images was obtained from the patient.

\section{Competing interests}

The authors declare that they have no competing interests.

\section{References}

1. Watanabe M, Tachimori $\mathrm{Y}$, Oyama $\mathrm{T}$, Toh $\mathrm{Y}$, Matsubara H, Ueno M, Kono K, Uno T, Ishihara R, Muro K, et al Comprehensive registry of esophageal cancer in Japan, 2013. Esophagus 18: 1-24, 2021.

2. Kitagawa Y, Uno T, Oyama T, Kato K, Kato H, Kawakubo H, Kawamura O, Kusano M, Kuwano $\mathrm{H}$, Takeuchi $\mathrm{H}$, et al: Esophageal cancer practice guidelines 2017 edited by the Japan esophageal society: Part 2. Esophagus 16: 25-43, 2019.

3. Huddy JR, Thomas RL, Worthington TR and Karanjia ND: Liver metastases from esophageal carcinoma: Is there a role for surgical resection? Dis Esophagus 28: 483-487, 2015.

4. Miyata H, Yamasaki M, Kurokawa Y, Takiguchi S, Nakajima K, Fujiwara Y, Konishi K, Mori M and Doki Y: Survival factors in patients with recurrence after curative resection of esophageal squamous cell carcinomas. Ann Surg Oncol 18: 3353-3361, 2011

5. Bleiberg H, Conroy T, Paillot B, Lacave AJ, Blijham G, Jacob JH, Bedenne L, Namer M, De Besi P, Gay F, et al: Randomised phase II study of cisplatin and 5-fluorouracil (5-FU) versus cisplatin alone in advanced squamous cell oesopahgeal cancer. Eur J Cancer 33: 1216-1220, 1997.

6. Iizuka $\mathrm{T}$, Kakegawa $\mathrm{T}$, Ide $\mathrm{H}$, Ando $\mathrm{N}$, Watanabe $\mathrm{H}$, Tanaka O, Takagi I, Isono K, Ishida K, Arimori M, et al: Phase II evaluation of cisplatin and 5-fluorouracil in advanced squamous cell carcinoma of the esophagus: A Japanese esophageal oncology group trial. Jpn J Clin Oncol 22: 172-176, 1992.

7. Hellman S and Weichselbaum RR: Oligometastases. J Clin Oncol 13: 8-10, 1995

8. Jamel S, Tukanova K and Markar S: Detection and management of oligometastatic disease in oesophageal cancer and identification of prognostic factors: A systematic review. World J Gastrointest Oncol 11: 741-749, 2019.

9. Li J, Wen Y, Xiang Z, Du H, Geng L, Yang X, Zhang Y, Bai J, Dai T, Feng G, et al: Radical radiotherapy for metachronous oligometastasis after initial treatment of esophageal cancer Radiother Oncol 154: 201-206, 2021.

10. Ohkura Y, Shindoh J, Ueno M, Iizuka T and Udagawa H: Clinicopathologic characteristics of oligometastases from esophageal cancer and long-term outcomes of resection. Ann Surg Oncol 27: 651-659, 2020.
11. Baba Y, Watanabe M, Kawanaka K, Iwagami S, Ishimoto T, Iwatsuki M, Yoshida N, Yamashita Y and Baba H: Radiofrequency ablation for pulmonary metastases from esophageal squamous cell carcinoma. Dis Esophagus 27: 36-41, 2014.

12. Dawood O, Mahadevan A and Goodman KA: Stereotactic body radiation therapy for liver metastases. Eur J Cancer 45: 2947-2959, 2009.

13. Lehrer EJ, Singh R, Wang M, Chinchilli VM, Trifiletti DM, Ost P, Siva S, Meng MB, Tchelebi L and Zaorsky NG: Safety and survival rates associated with ablative stereotactic radiotherapy for patients with oligometastatic cancer: A systematic review and meta-analysis. JAMA Oncol 7: 92-106, 2021.

14. Palma DA, Olson R, Harrow S, Gaede S, Louie AV, Haasbeek C, Mulroy L, Lock M, Rodrigues GB, Yaremko BP, et al: Stereotactic ablative radiotherapy for the comprehensive treatment of oligometastatic cancers: Long-term results of the SABR-COMET phase II randomized trial. J Clin Oncol 38: 2830-2838, 2020.

15. Pennathur A, Gibson MK, Jobe BA and Luketich JD: Oesophageal carcinoma. Lancet 381: 400-412, 2013.

16. Palma DA, Salama JK, Lo SS, Senan S, Treasure T, Govindan R and Weichselbaum R: The oligometastatic state - separating truth from wishful thinking. Nat Rev Clin Oncol 11: 549-557, 2014.

17. Morinaga T, Iwatsuki M, Yamashita K, Harada K, Kurashige J, Nagai Y, Iwagami S, Baba $\mathrm{Y}$, Yoshida $\mathrm{N}$ and Baba $\mathrm{H}$ : Oligometastatic recurrence as a prognostic factor after curative resection of esophageal squamous cell carcinoma. Surg Today 51: 798-806, 2021.

18. Rusthoven KE, Kavanagh BD, Cardenes H, Stieber VW, Burri SH, Feigenberg SJ, Chidel MA, Pugh TJ, Franklin W, Kane M, et al: Multi-institutional phase I/II trial of stereotactic body radiation therapy for liver metastases. J Clin Oncol 27: 1572-1578, 2009

19. Guckenberger M, Lievens Y, Bouma AB, Collette L, Dekker A, deSouza NM, Dingemans AC, Fournier B, Hurkmans C, Lecouvet FE, et al: Characterisation and classification of oligometastatic disease: A European society for radiotherapy and oncology and European organisation for research and treatment of cancer consensus recommendation. Lancet Oncol 21: e18-e28, 2020.

20. Ghaly G, Harrison S, Kamel MK, Rahouma M, Nasar A, Port JL, Stiles BM and Altorki NK: Predictors of survival after treatment of oligometastases after esophagectomy. Ann Thorac Surg 105: $357-362,2018$. 